\title{
Open Access Tubal aSsessment for the initial management of infertility in general practice (the OATS trial): a pragmatic cluster randomised controlled trial
}

\author{
Scott Wilkes, Alison Murdoch, Nick Steen, John Wilsdon and Greg Rubin
}

\begin{abstract}
Background

GPs investigate approximately half of all infertile couples with semen analysis and endocrine blood tests. For assessment of tubal status, hysterosalpingography (HSG) is recommended as a first-line investigation for women not known to have comorbidities.
\end{abstract}

Aim

To test whether providing GPs with open access to HSG results in infertile couples progressing to a diagnosis and management plan sooner than with usual management.

\section{Design of study}

A pragmatic cluster randomised controlled trial.

Setting

Seventy-one of 173 general practices in north-east England agreed to participate.

\section{Method}

A total of 670 infertile couples presented to 33 intervention practices and 25 control practices over a 2year period. Practices allocated to the intervention group had access to HSG for those infertile women who fulfilled predefined eligibility criteria. The primary outcome measure was the interval between presentation to the GP and the couple receiving a diagnosis and management plan.

\section{Results}

An annual incidence of 0.8 couples per 1000 total population equated to each GP seeing an average of one or two infertile couples each year. Open access HSG was used for $9 \%$ of all infertile women who presented to the intervention practices during the study period. The time to reach a diagnosis and management plan for all infertile couples presenting was not affected by the availability of open access HSG (Cox regression hazard ratio $=0.9$, $95 \%$ confidence interval $[\mathrm{Cl}]=0.7$ to 1.1 ). For couples who reached a diagnosis and management plan, there was a non-significant difference in time to primary outcome for intervention versus control practices $(32.5$ weeks versus 30.5 weeks, mean difference 2.2 weeks, $95 \% \mathrm{Cl}=1.6$ to 6.1 weeks, $P=0.1$ ). The intracluster correlation coefficient was 0.03 across all practices.

\section{Conclusion}

Providing GPs with open access to HSG had no effect on the time taken to reach a diagnosis and management plan for couples with infertility.

Keywords

family practice; health services accessibility; hysterosalpingography; infertility; primary health care; randomised controlled trial.

\section{INTRODUCTION}

Infertility affects approximately one in six couples during their lifetime, ${ }^{1,2}$ and a GP can expect to see one or two new infertile couples each year. ${ }^{1,3}$ GPs usually undertake an initial assessment of male fertility status and female ovulation status, with assessment of tubal patency being done under specialist care. In recent years the increasing regulation of fertility treatments has led to services being concentrated in dedicated tertiary care facilities. ${ }^{4}$ Referral of infertile couples to non-licensed units may delay access to definitive treatment. The inclusion of assisted reproduction in national 18week-to-treatment targets requires a more rapid investigation and referral pathway. Recent National Institute for Health and Clinical Excellence (NICE) guidelines on assessment and treatment for people with fertility problems ${ }^{5}$ includes a recommendation that hysterosalpingography (HSG) should be

S Wilkes, PhD, MRCGP, DRCOG, DFSRH, GP/clinical research fellow; G Rubin, FRCGP, professor, head of department and part-time GP, Centre for Primary and Community Care, School of Health Natural and Social Sciences, University of Sunderland, Sunderland. A Murdoch, $B S C, M D, F R C O G$, professor, consultant in reproductive medicine and head of department, Newcastle Fertility Centre at LIFE, BioScience Centre, International Centre for Life; $N$ Steen, PhD, senior statistician, Institute for Health and Society, University of Newcastle upon Tyne; J Wilsdon, FRCR, consultant radiologist and head of department, Department of Radiology, Royal Victoria Infirmary, Newcastle upon Tyne.

Address for correspondence

Dr Scott Wilkes, Centre for Primary and Community Care, School of Health Natural and Social Sciences, Priestman Building, Green Terrace, University of Sunderland, SR2 3PZ. E-mail: scott.wilkes@sunderland.ac.uk

Submitted: 9 August 2008; Editor's response: 27 October 2008; final acceptance: 19 December 2008.

(O)British Journal of General Practice 2009; 59: 329-335.

DOI: 10.3399/bjgp09X420590 


\section{How this fits in}

In the diagnostic process of infertility, GPs do not routinely have access to hysterosalpingography. However, women with tubal infertility identified on hysterosalpingography require referral for in vitro fertilisation or intracytoplasmic sperm injection at a Human Fertilisation and Embryology Authority-licensed tertiary care fertility unit. information leaflets for the HSG procedure. Written consent of couples entering the trial was obtained by their GPs (patient-level consent). Incident cases of infertile couples presenting to their GP between 31 December 2004 and 31 December 2006 were eligible to enter the trial. The general practice complete electronic clinical records including scanned correspondence from hospitals were the primary data source for the study.

considered as a first-line investigation for tubal status for women not known to have any comorbidities. $^{5}$ Direct access to HSG is recommended for the primary assessment of infertile couples on the NHS 18-week commissioning pathway website. ${ }^{6}$ This may contribute to a reduction in time from referral to definitive treatment. This research tested whether providing GPs with open access to HSG enabled the infertile couple to reach a diagnosis and management plan sooner than with usual management.

\section{METHOD}

\section{Setting and participants}

Seventy-one of the 173 practices (41\%) approached in the Newcastle upon Tyne, North Tyneside, South Tyneside, Gateshead, and Northumberland Primary Care Trust areas agreed to take part. All GP principals within the practice signed a statement of intent to participate in the study (cluster-level consent), ${ }^{7}$ and were invited to a local launch meeting where trial allocation was revealed and literature, including a one-page summary of the NICE guidance on infertility management, was provided. Intervention practices also received open access HSG and hospital radiology request forms as well as patient

\section{Box 1. Criteria for open access hysterosalpingography.}

The couple must have been trying to conceive for 1 year

- Normal semen analysis

- Normal coital function

- Female aged $<40$ years

Regular menstrual cycle ( $<6$ weeks)

Day 2-5 follicle-stimulating hormone $<10 \mathrm{IU} / \mathrm{I}$

- Mid-luteal progesterone $>20 \mathrm{nmol} / \mathrm{l}$

- No history of tubal disease

No known uterine or cervical abnormality

Negative endocervical swabs for chlamydia and gonococcus

- Rubella status checked

Female in receipt of folic acid

\section{Design}

This was a 1:1 cluster-randomised controlled trial with the practices as the unit of randomisation. Practices were stratified by geographical area. ${ }^{8} \mathrm{~A}$ permutated block design within each stratum ensured balanced allocation between intervention and control practices for large ( $>4$ whole-time equivalent [WTE] GPs), intermediate (2-4 WTE GPs), and small ( $<2$ WTE GPs) GP practices. Practices were randomly allocated, using SPSS (version 14.0), to intervention or control by a statistician with no prior knowledge of the practices.

\section{Intervention}

Intervention practices were able to request HSG through an open access service if their patients fulfilled predetermined eligibility criteria (Box 1). Use of the investigation remained at the discretion of the GP and the consenting woman. The open access service was provided at the radiology department of the Royal Victoria Infirmary, Newcastle upon Tyne (for Northumberland, Newcastle, North Tyneside) and at the Queen Elizabeth Hospital, Gateshead (for Gateshead and South Tyneside). In the control practices GPs did not have access to HSG, and following referral the investigation was traditionally requested by fertility specialists.

\section{Objective}

The study objective was to evaluate the effect of providing GPs with open access to HSG on time to diagnosis and completeness of the initial assessment for infertile couples. The latter was defined by the NICE recommendation which was embedded within the open access HSG criteria (Box 1).

\section{Outcomes}

The primary outcome was the time taken from presentation to the GP and the couple receiving a definitive cause and management plan for their infertility from a fertility specialist or their GP. A management plan was defined by the offer of treatment, whether ovulation induction with clomifene, in vitro fertilisation, intracytoplasmic sperm injection, donor eggs or sperm, weight loss, 


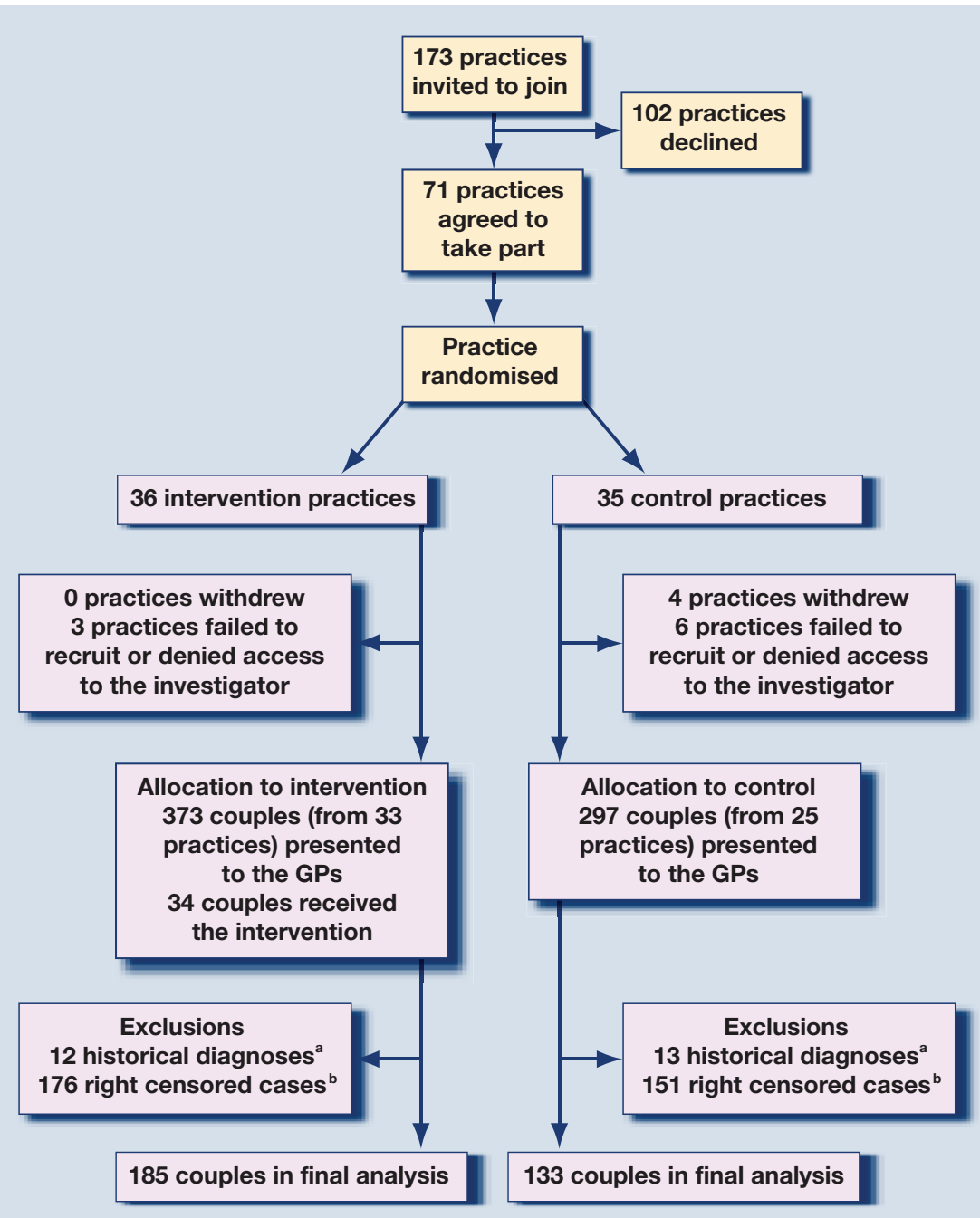

a25 couples excluded. This is a known study bias discussed in the Results section. A total of 327 couples did not reach the primary outcome measure (time from presentation to GP, to reaching a diagnosis and management plan) due to either spontaneous pregnancy, still being under investigation, or withdrawing from management.

intrauterine insemination, or reversal of sterilisation, or no treatment offered. Couples with no record of a diagnosis and/or management plan were considered not to have reached the primary outcome. A secondary outcome was the completeness of the initial assessment for infertile couples.

\section{Sample size}

Assumptions were that the data were normally distributed with similar standard deviations, that an average practice of three GPs would see six couples in 1 year, ${ }^{1}$ and that $50 \%$ of all infertile couples would be recruited to the trial, with approximately $50 \%$ eligible for open access HSG, giving an estimated 1.5 HSG referrals from each practice, that is a $25 \%$ uptake of the intervention. The estimated time to diagnosis and management plan for controls was 26 weeks (standard deviation
[SD] 14 weeks). ${ }^{3}$ With a best estimate for the intracluster correlation coefficient of $0.01,{ }^{9,10}$ to detect a $30 \%$ ( 26 weeks to 18 weeks) reduction in primary outcome with $80 \%$ power and $5 \%$ significance level, 36 practices (54 couples or 216 assuming $25 \%$ uptake) would be required in each arm of the trial.

\section{Statistical methods}

Data were analysed using SPSS and MLwiN. A survival analysis using a Cox proportional hazards model ${ }^{11}$ was used to account for couples that had not reached the primary outcome measure. For couples that reached the primary outcome measure, a cluster analysis using multilevel modelling (MLwiN) was employed. ${ }^{12}$ The effect of clustering was quantified using patient- and practice-level variances and presented as the intracluster correlation coefficient for the primary outcome measure.
Figure 1. Practices and participant flow. 
Table 1. Characteristics of the trial participants.

\begin{tabular}{lccc} 
& Intervention & Control & Total \\
\cline { 2 - 4 } & $n=373$ & $n=297$ & $n=670$ \\
\hline Age of female: years, mean (SD) & $30(6)$ & $31(6)$ & $31(6)$ \\
\hline Trying to conceive: months, mean (SD) & $24(15)$ & $23(15)$ & $23(15)$ \\
\hline Primary infertility: female, $n(\%)$ & $207(56)$ & $175(59)$ & $382(57)$ \\
\hline Couple fertility status, $n(\%)$ & & & \\
Primary infertility & & & \\
Secondary infertility & $171(46)$ & $150(51)$ & $321(48)$ \\
Tertiary infertility & $104(28)$ & $82(28)$ & $186(28)$ \\
$\quad$ Female primary and male secondary & & & \\
$\quad$ Female secondary and male primary & $35(9)$ & $26(9)$ & $61(9)$ \\
$\quad$ Both secondary, other union & $30(8)$ & $21(7)$ & $51(8)$ \\
\hline Referred by GP: total, $n$ (\%) & $31(8)$ & $18(6)$ & $49(7)$ \\
$\quad$ To tertiary care NHS,$n$ (\% of total referred) & $266(71)$ & $213(72)$ & $479(72)$ \\
To secondary care NHS,$n$ (\% of total referred) & $116(36)$ & $116(39)$ & $251(37)$ \\
Privately & $15(4)$ & $85(29)$ & $201(30)$ \\
\hline
\end{tabular}

aPrimary infertility: having never had a child following 12 months' unprotected intercourse. 'Secondary infertility: difficulty conceiving after already having a child. 'Tertiary infertility: difficulty conceiving after one or both partners already have a child in a previous union. ${ }^{\circ}$ Tertiary care: fertility services licensed by the Human Fertilisation and Embryology Authority to delivery specialist services including in vitro fertilisation and intracytoplasmic sperm injection. eSecondary care: general gynaecology services not licensed by the Human Fertilisation and Embryology Authority.

Table 2. Information recorded on GP medical record and referral letter; number (\%).

\begin{tabular}{lcccc} 
& \multicolumn{2}{c}{ Intervention } & \multicolumn{2}{c}{ Control } \\
\cline { 2 - 5 } & GP record & Referral letter & GP record & Referral letter \\
\cline { 2 - 5 } & $n=373$ & $n=266$ & $n=297$ & $n=213$ \\
\hline Information recorded & & & & \\
Length of time trying & $373(100)$ & $205(77)$ & $297(100)$ & $141(66)$ \\
Fertility status $^{\mathrm{a}}$ & $373(100)$ & $168(63)$ & $297(100)$ & $93(44)$ \\
Cycle status recorded $^{2}$ & $281(75)$ & $144(54)$ & $196(66)$ & $103(48)$ \\
<42-week cycle $236(63)$ & $115(43)$ & $173(58)$ & $87(41)$ \\
Folate supplementation & $150(40)$ & $41(15)$ & $93(31)$ & $19(9)$ \\
Recorded comorbidities $^{\mathrm{b}}$ & $78(21)$ & $44(17)$ & $56(19)$ & $35(16)$ \\
Smoking status recorded & $59(16)$ & $23(9)$ & $37(12)$ & $17(8)$ \\
Alcohol status recorded & $42(11)$ & $15(6)$ & $24(8)$ & $10(5)$ \\
Frequency of intercourse & $35(9)$ & $7(3)$ & $23(8)$ & $9(4)$ \\
Information on tubal status & $18(5)$ & $14(5)$ & $9(3)$ & $7(3)$ \\
Testicular advice given & $6(2)$ & $3(1)$ & 0 & 0 \\
Advice regarding drugs & $3(1)$ & 1 & 1 & 1 \\
\hline Investigations recorded & & & & \\
Mid-luteal progesterone & $258(69)^{\mathrm{c}}$ & $149(56)$ & $208(70)^{\circ}$ & $112(53)$ \\
FSH/LH & $220(59)^{\mathrm{d}}$ & $113(42)$ & $168(57)^{\mathrm{d}}$ & $88(41)$ \\
Semen analysis & $200(54)^{\mathrm{e}}$ & $120(45)$ & $152(51)^{\mathrm{e}}$ & $92(43)$ \\
Thyroid function tests & $172(46)$ & $72(27)$ & $154(52)$ & $64(30)$ \\
Full blood count & $153(41)$ & $61(23)$ & $111(37)$ & $44(21)$ \\
Prolactin & $92(25)$ & $39(15)$ & $70(24)$ & $30(14)$ \\
\hline Rubella & $75(20)$ & $38(14)$ & $60(20)$ & $27(13)$ \\
\hline Random blood glucose & $58(16)$ & $21(8)$ & $40(13)$ & $16(8)$ \\
\hline Chlamydia & $62(17)$ & $27(10)$ & $23(8)$ & $7(3)$ \\
\hline Oestrogen & $13(3)$ & $6(2)$ & $14(5)$ & $7(3)$ \\
\hline Testosterone & $40(11)$ & $12(5)$ & $21(7)$ & $11(5)$ \\
\hline Other & $79(21)$ & $12(5)$ & $58(20)$ & $18(8)$ \\
\hline
\end{tabular}

aPrimary, secondary, or tertiary infertility. ${ }^{\circ}$ Obesity $28 \%$, polycystic ovary syndrome $25 \%$, tubal pathology $22 \%$, vasectomy $10 \%$, chlamydia $5 \%$, other $10 \%$. 'Adjusted odds ratio (OR) $=1.1, \mathrm{P}=$ 0.3. ${ }^{\circ}$ Adjusted $\mathrm{OR}=1.1, \mathrm{P}=0.3$. ${ }^{\circ}$ Adjusted $\mathrm{OR}=1.1, \mathrm{P}=0.3$. Other investigations included urea and electrolytes, liver function tests, sex hormone-binding globulin, and ultrasound scan. FSH = follicle-stimulating hormone. $\mathrm{LH}=$ luteinising hormone.
Adjusted mean differences between groups are presented with $95 \%$ confidence intervals $(\mathrm{Cl})$, and $P<0.05$ was considered significant.

Secondary analyses on the primary outcome were adjusted for the effect of geographical area, use of open access HSG, and place of referral.

Secondary outcomes were a measure of the uptake of open access HSG and a logistic regression model for binomial response data (investigations performed in both groups), adjusting for patient clustering.

\section{RESULTS}

\section{Participant flow}

There were 670 incident couples over the 2-year period from 58 practices (Figure 1), with a combined list size of 403263 patients (72 182 being women aged 16-42 years). This represents an annual incidence of 0.8 infertile couples per 1000 total population, or 4.6 couples per 1000 women aged 16-42 years. Characteristics of the trial participants are shown in Table 1.

Initial investigations recommended by the NICE guideline, that is, semen analysis, mid-luteal progesterone, and follicle-stimulating hormone (FSH), were done for between half and two-thirds of couples. Of those couples referred (479/670), GPs were most efficient at passing on information about the length of time a couple were trying to conceive, their fertility status, menstrual cycle status, and midluteal progesterone result (Table 2).

\section{Primary outcome}

The time from first presentation to diagnosis and management plan comprises three elements: time from presentation to the GP to being referred, time from referral to being seen by the fertility specialist, and time from being seen by the fertility specialist to the couple receiving a diagnosis and management plan. In this study these intervals were 7.1 weeks $(\mathrm{SD}=9.7), 9.7$ weeks $(\mathrm{SD}=4.4)$, and 14.3 weeks (SD = 13.6) respectively. Three per cent (11/343) of couples were given a diagnosis and management plan by their GP.

For all couples ( $n=670$ ), a Kaplan-Meier survival analysis and Cox regression/proportional hazards model (hazard ratio $0.9,95 \% \mathrm{Cl}=0.7$ to $1.1, P=0.3$ ) demonstrated a non-significant difference between intervention and control couples reaching the primary outcome (Figure 2). A definitive diagnosis and management plan was reached by $343(51 \%)$ couples (Table 3). Twenty-five couples (12 from intervention and 13 from control) who had an historical diagnosis were excluded, leaving 318 couples (133 control and 185 intervention) in the final analysis. 


\section{Multilevel modelling on couples reaching outcome ( $n=318$ couples)}

The intracluster correlation coefficient was 0.03 across all practices. There was a non-significant difference in time to primary outcome for intervention versus control practices (32.5 versus 30.3 weeks, mean difference 2.2 weeks, $95 \% \mathrm{Cl}=-1.6$ to 6.1 weeks, $P=0.1$.

\section{Secondary analyses on the primary outcome: factors influencing time to definitive diagnosis and management plan}

There were significant differences between areas; the average time to definitive diagnosis and management plan varied from 27.8 weeks (Gateshead) to 34.8 weeks (Northumberland).

Thirty-four couples underwent open access HSG. Mean time to primary outcome was 22.0 weeks compared to 32.5 weeks for couples that did not have open access HSG (mean difference -10.5 weeks, $95 \% \mathrm{Cl}=-16.4$ to -4.6 weeks, $P<0.001)$. Two-thirds of couples that had open access HSG performed (23/34) were subsequently referred. For all couples referred in the trial $(n=479)$, the completeness of information passed on in the referral letter was greater for patients who had undergone HSG (Table 4); however, this was not statistically significant given the small numbers in the open access HSG group. When compared with referrals to tertiary fertility units, referrals to secondary care took 8.7 weeks longer $(95 \% \mathrm{Cl}=5.2$ to 12.2 weeks) to reach the primary outcome measure, but couples referred privately reached the outcome 8.3 weeks sooner $(95 \% \mathrm{Cl}=-17.7$ to 0.8 weeks).

\section{Secondary outcomes}

The uptake of open access HSG in the intervention practices was 9\% (34/361). Intervention practices were no more likely than control practices to perform the initial investigations recommended by NICE guidance; semen analysis (adjusted odds ratio [OR] $1.1,95 \% \mathrm{Cl}=-0.5$ to $2.7, P=0.3)$, mid-luteal progesterone (adjusted OR $=1.1,95 \% \mathrm{Cl}=-0.5$ to 2.7, $P=0.3$ ), and serum FSH (adjusted $\mathrm{OR}=1.1$, $95 \% \mathrm{Cl}=-0.3$ to $2.7, P=0.3$ ); (Table 2 ).

\section{DISCUSSION}

\section{Summary of main findings}

Making HSG available to GPs did not decrease the time taken for couples to reach a definitive diagnosis and management plan. Nine per cent of couples in the intervention arm of the study were referred for open access HSG, and these couples reached a diagnosis and management plan 12 weeks earlier compared with usual management. One-third of

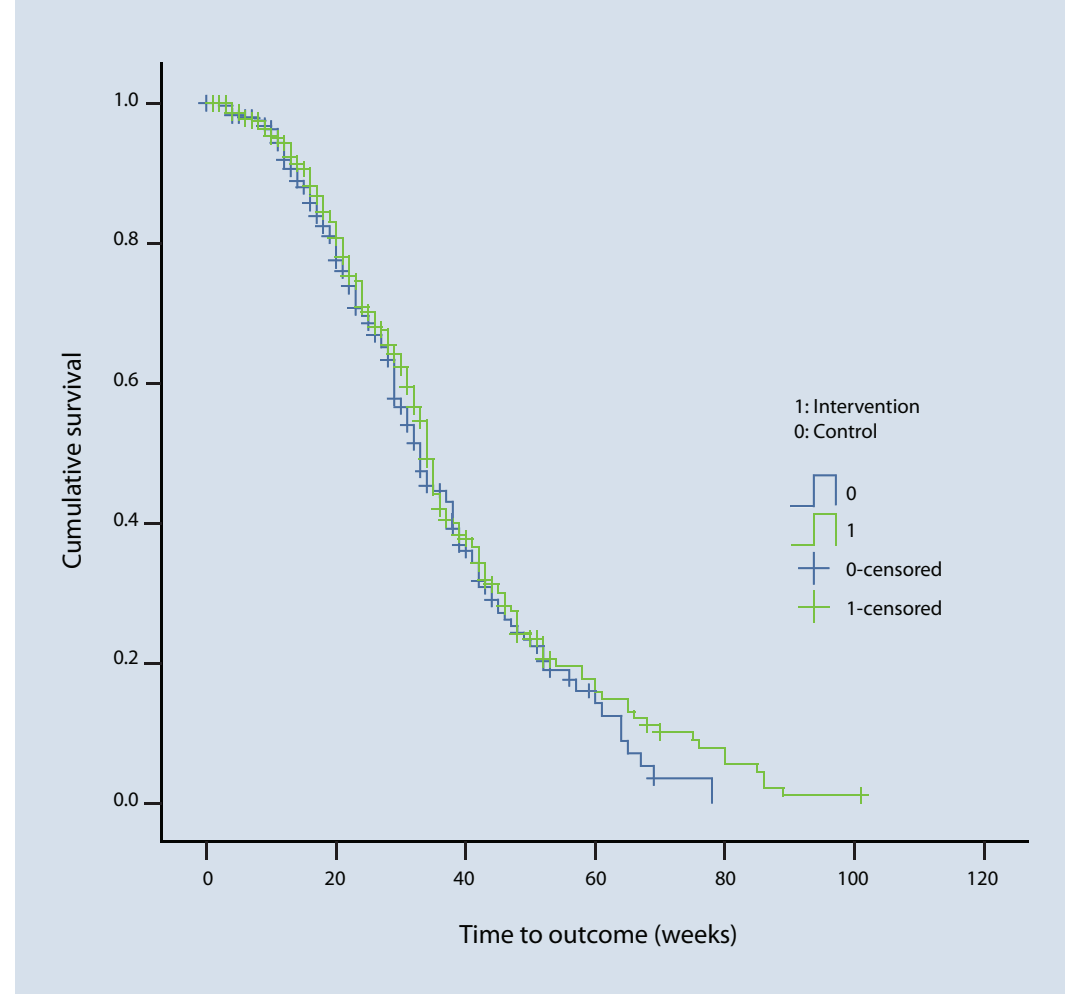

couples that had open access HSG performed were not subsequently referred as the GPs adopted a process of 'watchful waiting' with a diagnosis of unexplained infertility. For couples that had open access HSG performed, the guidance on initial investigations embedded within the form increased the likelihood that they were completed. Only $3 \%$ of the variation in primary outcome can be explained by clustering within practices, with most of the variation occurring at the patient level, showing that there is consistency of fertility management between practices.

\section{Strengths and limitations of the study}

This was a pragmatic trial that gives a measure of the usefulness of open access HSG in usual clinical practice. By randomising practices rather than individual GPs, contamination bias and selection bias were minimised. However, a high dropout rate

Table 3. Outcomes of infertile couples presenting to GPs; number (\%).

\begin{tabular}{lccc} 
& Intervention & Control & Total \\
\cline { 2 - 4 } & $n=373$ & $n=297$ & $n=670$ \\
\hline Reached primary outcome measure $^{\mathrm{a}}$ & $197(53)$ & $146(49)$ & $343(51)$ \\
\hline Spontaneous pregnancy & $68(18)$ & $70(24)$ & $138(21)$ \\
\hline Incomplete investigations & $74(20)$ & $63(21)$ & $137(20)$ \\
\hline Withdrew from investigations & $34(9)$ & $18(6)$ & $52(8)$ \\
\hline
\end{tabular}

${ }^{a} 12$ couples in intervention and 13 couples in control had historical diagnoses, hence reached the outcome immediately upon consultation. 


\begin{tabular}{|c|c|c|}
\hline & $\begin{array}{l}\text { Referrals following } \\
\text { open access HSG }\end{array}$ & $\begin{array}{l}\text { Referred directly } \\
\text { to hospital }\end{array}$ \\
\hline & $n=23$ & $n=456$ \\
\hline \multicolumn{3}{|l|}{ Advice } \\
\hline Frequency of sexual intercourse & $2(9)$ & $14(3)$ \\
\hline Avoiding testicular hyperthermia & 0 & $3(0)$ \\
\hline Smoking & $3(13)$ & $37(8)$ \\
\hline Alcohol & $1(4)$ & $24(5)$ \\
\hline Recreational drug use & 0 & $2(0)$ \\
\hline \multicolumn{3}{|l|}{ Information } \\
\hline Comorbidities & $2(9)$ & $77(17)$ \\
\hline Rubella status & $10(43)$ & $55(12)$ \\
\hline In receipt of folic acid & $14(61)$ & $46(10)$ \\
\hline \multicolumn{3}{|l|}{ Investigations } \\
\hline Semen analysis & $23(100)$ & $189(41)$ \\
\hline Mid-luteal progesterone & $23(100)$ & $238(52)$ \\
\hline Follicle stimulating hormone & $22(96)$ & $179(39)$ \\
\hline Thyroid function test & $6(26)$ & $130(29)$ \\
\hline Prolactin & $6(26)$ & $63(14)$ \\
\hline
\end{tabular}

of control practices may have contributed to a postrandomisation selection bias. Stratification minimised biases due to differential access and referral patterns. Following practice randomisation, differential recruitment within intervention and control clusters remained an ever present threat. There was low uptake of open access HSG by the intervention practices, which diluted its effect size. The secondary per protocol 'on treatment' analysis was underpowered and biased. Those that received open access HSG and were subsequently referred, not surprisingly, had better information transferred in the referral letter, which probably reflected individual GP interest in fertility management.

\section{Comparison with existing literature}

The incidence (one couple per 1000 population) $)^{1,13}$ and management of infertility in primary care has remained largely unchanged over the last 10 years. ${ }^{3,14-16}$ One survey has shown that $60-80 \%$ of GPs agree that the initial investigations recommended by the Royal College of Obstetricians and Gynaecologists and subsequently by NICE should be carried out in primary care. ${ }^{17}$ Clinical guidelines alone, however, are generally insufficient to influence GPs' behaviour and referral patterns, ${ }^{18-21}$ and the implementation of guidelines should be tailored to identify potential barriers. ${ }^{22}$ Two studies on infertility guidelines have shown a modest improvement in GP referral patterns, ${ }^{23,24}$ although this change in behaviour was not sustained. ${ }^{15}$ Embedding clinical guidelines within the open access referral process has been shown to increase compliance with referral standards and improved service efficiency for open access gastroscopy and open access urological investigations. ${ }^{25,26}$ The uptake of open access HSG was low in this study, with only $9 \%$ of couples being referred. Data that became available during this study suggested that $26 \%$ of couples presenting to GPs could be eligible for open access HSG based on this study's criteria. ${ }^{27}$

\section{Implications for future research and clinical practice}

Some GPs have shown a willingness to use criteriadriven open access HSG, enabling a management plan to be established at the first specialist consultation. In this study, 9\% of couples benefited from open access HSG and reduced the duration of their 'patient journey' from presentation to the GP to specialist treatment, although this still fell short of the government 18 -week target. ${ }^{28}$ If open access HSG is introduced for GPs, future research will need to assess its impact on the whole patient journey, referral patterns, and cost-effectiveness.

\section{Funding body}

We would like to acknowledge the NHS National Coordinating Centre for Research Capacity Development who funded this work through a National Institute for Health Research (NIHR) award held by Dr Scott Wilkes (RDA03/26); Primary Care Researcher Development Award, National Coordinating Centre for Research Capacity Development, Leeds Innovation Centre. All researchers involved in the production of this paper are independent from the funder. The trial is Current Controlled Trials ISRCTN18398372

\section{Ethics committee}

Ethical approval was granted from Newcastle and North Tyneside as the main REC with SSA approval from Northumberland, Gateshead, and South Tyneside Local Research Ethics Committees. Approval was also granted from Sunderland University Research Ethics Committee

\section{Competing interests}

The authors have stated that there are none

\section{Acknowledgements}

Academics within Sunderland University contributed to the study design; collection, analysis, and interpretation of data; production of the paper and the decision to submit the article for publication.

\section{Discuss this article}

Contribute and read comments about this article on the Discussion Forum: http://www.rcgp.org.uk/bjgp-discuss

\section{REFERENCES}

1. Hull MG, Glazener CM, Kelly NJ, et al. Population study of causes, treatment, and outcome of infertility. Br Med J (Clin Res Ed) 1985; 291(6510): 1693-1697.

2. Templeton A, Fraser C, Thompson B. The epidemiology of infertility in Aberdeen. BMJ 1990; 301(6744): 148-152.

3. Wilkes S, Jones K. Retrospective review of the prevalence and management of infertility in women in one practice over a five year period. Br J Gen Pract 1995; 45(391): 75-77.

4. Human Fertilisation and Embryology Authority Newsletter. Should embryo screening help parents prevent passing on a wider range of inheritable diseases? http://www.hfea.gov.uk/docs/HFEA_Update_ Nov_05.pdf (accessed 6 Apr 2009).

5. National Institute for Clinical Excellence. Fertility: assessment and treatment for people with fertility problems. London, National Institute for Clinical Excellence, 2004; 1-45. 
6. Department of Health. Delivering the 18 week patient pathway. http://www.18weeks.nhs.uk (accessed 20 January 2009).

7. Campbell MK, Grimshaw JM. Cluster randomised trials: time for improvement. The implications of adopting a cluster design are still largely being ignored. BMJ 1998; 317(7167): 1171-1172.

8. Medical Research Council. Cluster randomised trials: methodological and ethical considerations. London: Medical Research Council, 2002.

9. Adams G, Gulliford MC, Ukoumunne OC, et al. Patterns of intracluster correlation from primary care research to inform study design and analysis. J Clin Epidemiol 2004; 57(8): 785-794.

10. Campbell MK, Mollison J, Grimshaw JM. Cluster trials in implementation research: estimation of intracluster correlation coefficients and sample size. Stat Med 2001; 20(3): 391-399.

11. Bland M. An introduction to medical statistics. Oxford: Oxford University Press, 1996.

12. Campbell MK, Mollison J, Steen N, et al. Analysis of cluster randomized trials in primary care: a practical approach. Fam Pract 2000; 17(2): 192-196.

13. Oakley L, Doyle P, Maconochie N, et al. Lifetime prevalence of infertility and infertility treatment in the UK: results from a population-based survey of reproduction. Hum Reprod 2008; 23(2): $447-450$.

14. Das S, Chin KA. A study of pre-referral evaluation of infertile couples. J Obstet Gynaecol 2003; 23(1): 70.

15. Morrison C, Bhattacharya S, Hamilton M, et al. Initial management of infertility: an audit of pre-referral investigations and exploration of couples' views at the interface of primary and secondary care. Hum Fertil 2007; 10(1): 25-31.

16. Nicopoullos JD, Croucher CA. Audit of primary care and initia secondary care investigations set against RCOG guidelines as standard in cases of subfertility. J Obstet Gynaecol 2003; 23(4): 397-401.

17. Souter VL, Penney G, Gorman DR. A survey of infertility practices in primary care in Scotland. Br J Gen Pract 1997; 47(424): 727-728.

18. Grimshaw J, Shirran E, Thomas R, et al. Getting evidence into practice. Eff Health Care 1999; 5: 1-16.

19. Grol R. Personal paper. Beliefs and evidence in changing clinical practice. BMJ 1997; 315(7105): 418-421.

20. Robertson N, Baker R, Hearnshaw H. Changing the clinical behavior of doctors: a psychological framework. Qual Health Care 1996; 5(1): 51-54

21. Wensing $M$, van der Weijden T, Grol R. Implementing guidelines and innovations in general practice: which interventions are effective? Br J Gen Pract 1998; 48(427): 991-997.

22. Bosch $\mathrm{M}$, van der Weijden $\mathrm{T}$, Wensing $\mathrm{M}$, et al. Tailoring quality improvement interventions to identified barriers: a multiple case analysis. J Eval Clin Pract 2007; 13(2): 161-168.

23. Emslie C, Grimshaw J, Templeton A. Do clinical guidelines improve general practice management and referral of infertile couples? BMJ 1993; 306(6894): 1728-1731.

24. Morrison J, Carroll L, Twaddle S, et al. Pragmatic randomised controlled trial to evaluate guidelines for the management of infertility across the primary care-secondary care interface. $B M J$ 2001; 322(7294): 1282-1284.

25. Thomas RE, Grimshaw JM, Mollison J, et al. Cluster randomized trial of a guideline-based open access urological investigation service. Fam Pract 2003; 20(6): 646-654

26. Zuccaro GJ, Provencher K. Does an open access system properly utilize endoscopic resources? Gastrointest Endosc 1997; 46(1): 15-20.

27. Wilkes S, Murdoch A, Rubin G, et al. Investigation of infertility management in primary care with open access hysterosalpingography (HSG): a pilot study. Hum Fertil 2006; 9(1): $47-51$.

28. Department of Health. The NHS improvement plan: putting people at the heart of public services. London: Department of Health, 2004. 